\title{
Pomeranian atRial flOw reguLatOr iN conGestive hEart failuRe (PROLONGER): Study protocol
}

\author{
Łukasz Lewicki ${ }^{1,3}$, Katarzyna Kosmalska ${ }^{2}$, Sebastian Liedtke ${ }^{3}$, \\ Maciej Karwowski ${ }^{3}$, Janusz Siebert ${ }^{1,4}$, Robert Sabiniewicz ${ }^{5}$, \\ Jakub Kiedrzyn ${ }^{3}$, Adrian Kot ${ }^{3}$, Marek Szołkiewicz ${ }^{3}$ \\ ${ }^{1}$ University Center for Cardiology, Gdansk, Poland \\ ${ }^{2}$ Department of Cardiology, Pomeranian Hospitals, Gdynia, Poland \\ ${ }^{3}$ Department of Cardiology and Angiology, Kashubian Center for Heart and Vascular Diseases, \\ Pomeranian Hospitals, Wejherowo, Poland \\ ${ }^{4}$ Department of Family Medicine, Medical University of Gdansk, Poland \\ ${ }^{5}$ Department of Pediatric Cardiology and Congenital Heart Disease, Medical University of Gdansk, Poland
}

\section{Background}

Heart failure (HF) remains as important challenge in cardiovascular medicine. The incidence increases with age reaching $10 \%$ of the human population after the seventh decade of life $[1,2]$. Despite advances in HF therapy, morbidity remains high regardless of HF etiology. Treatment is focused mainly on the reduction of symptoms; however, it has been shown to date that a reduction in mortality was achieved only in heart failure patients with reduced ejection fraction (HFrEF) [3]. On top of that, the group of patients with heart failure with preserved ejection fraction $(\mathrm{HFpEF})$ continues to grow.

The diastolic dysfunction, which is common among HFpEF patients is mainly driven by diminished left ventricle relaxation, increased volume and filling pressures in the left atrium that leads to pulmonary congestion $[4,5]$. In these patients, treatment options are limited mainly to diuretics.

A novel conception of therapy based on creating a communication between both atria has been proposed recently. The left to right interatrial shunt enables decompression of the left atrium and thus may improve patient symptoms $[6,7]$.

There are three different devices available for patients with either HFrEF or HFpEF: interatrial shunt device (IASD, Corvia Medical Inc., Tewksbury, MA, USA); V-Wave shunt (V-Wave Ltd.,
Caesarea, Israel) and the Atrial Flow Regulator (AFR, Occlutech, Heslingborg, Sweden). The initial safety and efficacy have been proved for the first two devices in small studies [6, 7]. Atrial Flow Regulator is a self-expandable double-disc nitinol wire mesh construction allowing communication across the interatrial septum. The offered fenestration diameter ranges from $4 \mathrm{~mm}$ to $10 \mathrm{~mm}$, but for $\mathrm{HF}$ patients only the $8 \mathrm{~mm}$ and $10 \mathrm{~mm}$ have the European CE mark. The device is fully repositionable and retrievable.

There are few data concerning the effectiveness of interatrial shunting. It is reasonable to focus on defining clinical and hemodynamic parameters, thus predicting who would benefit from interatrial shunting. The Pomeranian atRial flOw reguLatOr iN conGestive hEart failuRe (PROLONGER) study was designed for this purpose.

\section{Methods}

\section{Study design \\ The PROLONGER study (ClinicalTrials.gov identifier NCT04334694) is a prospective, open- -label clinical trial aimed to define invasive and non- invasive parameters that could predict a positive response for AFR therapy among patients with HF. The observation period will include peri-procedural hospitalization and a 12 month follow-up with four con- trol visits: after 1, 3, 6 and 12 months, respectively.}

Address for correspondence: Łukasz Lewicki, MD, PhD, University Center for Cardiology, ul. Dębinki 2, 80-211 Gdańsk, Poland, tel: +48501 702 885, e-mail: luklewicki@gmail.com 


\section{Patients' selection}

Around 30 patients will be enrolled. The main inclusion criterion is symptomatic $\mathrm{HF}$, either HFrEF or HFpEF. An elevated left heart filling pressure has to be confirmed in all eligible patients before the AFR procedure. Detailed inclusion and exclusion criteria are presented in Table 1.

\section{Blinding (masking)}

As the PROLONGER is an open-label, single-arm study, both the participants and investigators will not be blinded to the allocated intervention.

\section{Interventions}

All eligible patients will undergo detailed screening using echocardiography, impedance cardiography (ICG) and right heart catheterization prior to AFR implantation.

Echocardiography. The transthoracic echocardiography (TTE) examinations will be taken as recommended by European Society of Cardiology (ESC) and American Heart Association (AHA). Patients will be imaged in the left lateral decubitus position. Images will be obtained in the parasternal and apical chamber views.

The echocardiography protocol consists of the following elements recorded with three cardiac cycles loop recordings:

- Long axis view (LAX): Dimensions of left and right ventricles, left atrium, morphology and function of aortic and mitral valves;

- Short axis view (SAX): Morphology and function of aortic and pulmonic valves; pulse wave Doppler in right ventricle outflow tract (RVOT) (assessment of acceleration time);

- Apical 4 and $2 \mathrm{C}$ plane:

- Assessment of right ventricle dimension right ventricle inflow tract (RVIT), right atrium area, left atrium volume index (LAVI),

- Assessment of left ventricle systolic function (ejection fraction by Simpson method and by speckle tracking imaging),

- Assessment of left ventricle diastolic function (by using mitral flow, mitral annulus diastolic velocity, left atrial volume, tricuspid regurgitation (TR) velocity,

- Assessment of right ventricle systolic function by tricuspid annular plane systolic excursion (TAPSE), right ventricle fractional area change and speckle tracking imaging,

- Morphology and function of mitral valve,
Table 1. The inclusion and exclusion criteria in PROLONGER study.

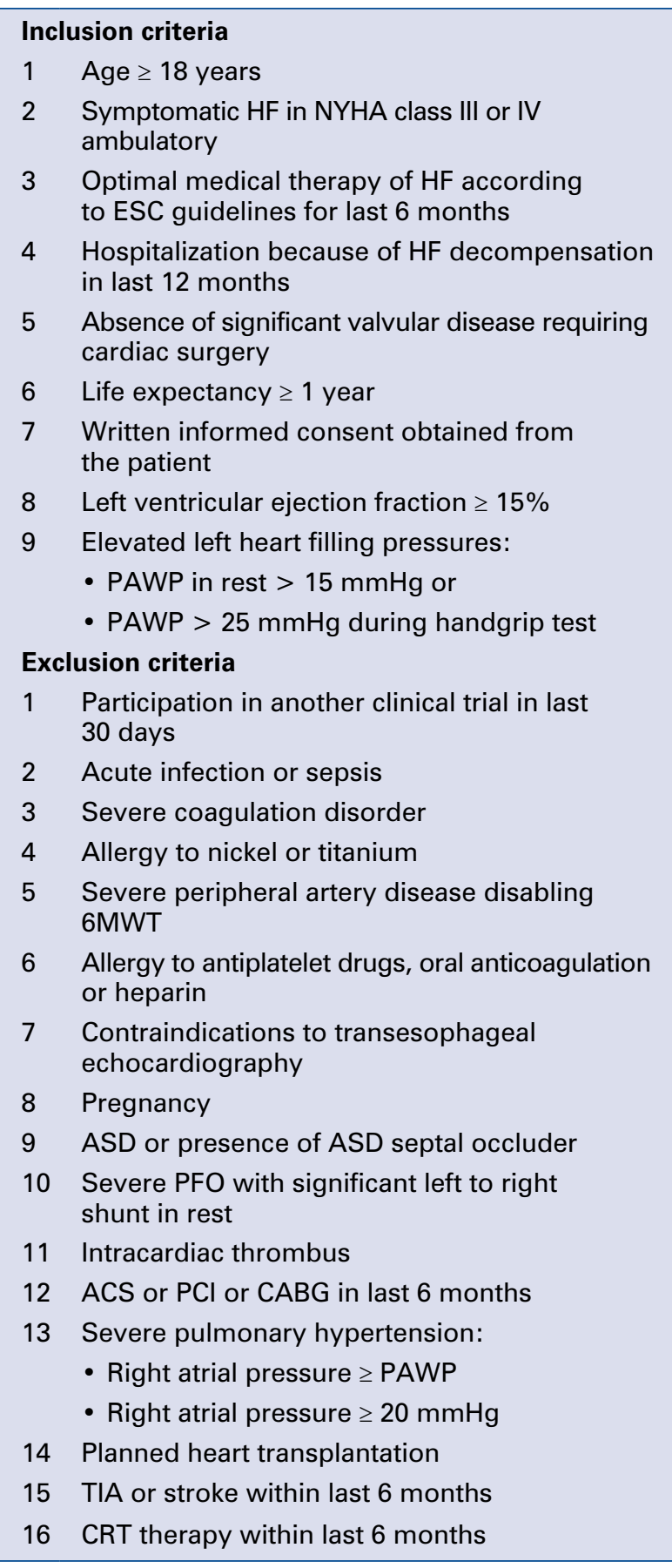

HF — heart failure; NYHA — New York Heart Association; ESC - European Society of Cardiology; PAWP — pulmonary artery wedge pressure; 6MWT — six-minute walk test; ASD — atrial septal defect; PFO - patent foramen ovale; ACS - acute coronary syndrome; $\mathrm{PCl}$ - percutaneous coronary intervention; CABG coronary artery bypass grafting; TIA — transient ischemic attack; $\mathrm{CRT}$ - cardiac resynchronization therapy

- Mechanism and quantitative assessment of mitral regurgitation,

- Morphology and function of aortic valve, 
- Morphology and function of tricuspid valve,

- Quantitative assessment of tricuspid regurgitation;

- Subcostal view:

- Position and flow (color Doppler) in AFR device, flow gradient (continuous wave Doppler).

The TTE examinations will be done before AFR and during each follow up visit.

All patients will also be imaged with transesophageal echocardiography (TEE) in standard views to:

- Exclude intra-cardiac thrombus;

- Exclude presence of patent foramen ovale (PFO) or atrial septal defect (ASD);

- Assess anatomy of interatrial septum.

The TEE examinations will be done prior to AFR procedure and during the first follow up visit.

Impedance cardiography. All patients will be examined using an ICG.

The PhysioFlow ${ }^{\circledR}$ Q-LinkTM is a noninvasive hemodynamic evaluation system to assess patient cardiovascular state using the analysis of transthoracic bio-impedance signals.

PhysioFlow System measures the change in impedance by injecting a high frequency alternating electrical current $(66 \mathrm{kHz})$ of low magnitude (4.5 $\mathrm{mA}$ peak to peak) towards the thorax between two electrodes positioned on the neck and another two positioned on xiphoïd process. The use of a high frequency current eliminates the risk of interference with heart and brain bioelectrical activity. In addition, as the impedance of skinelectrodes is very low at high frequency, tissues will not endure any thermal effects and patients feels nothing.

Specific calculations conducted, as part of further analysis will be based on values obtained in the supine position.

The measurement scheme will be as follows: the first ICG will be done 1 day prior to the AFR procedure. Four ICG and two ECG pre-gelled electrodes will be placed: two $(Z 1, Z 2)$ on the left side of the patient's neck, one (Z3) at the level of the xiphoid and the last one (Z4) just to the right to Z3. The ICG data will be collected continuously (beat by beat) for $30 \mathrm{~min}$. The blood pressure will be taken every $10 \mathrm{~min}$ and stored for analysis. The following parameters will be measured continuously: cardiac index (CI $\left[1 / \mathrm{min} / \mathrm{m}^{2}\right]$ ), stroke volume index $\left(\mathrm{SVi}\left[\mathrm{mL} / \mathrm{m}^{2}\right]\right)$, left cardiac work index (LCWi $\left[\mathrm{kg} \cdot \mathrm{m} / \mathrm{m}^{2}\right]$ ), contractility index (CTi), ventricular ejection time (VET [ms]), systemic vascular resistance index (SVRi $\left.\left[\mathrm{dyn} \cdot \mathrm{s} / \mathrm{cm}^{5} \cdot \mathrm{m}^{2}\right]\right)$, thoracic fluid content index (TFCi $\left[1 / \mathrm{k} \Omega \cdot \mathrm{m}^{2}\right]$ ) and heart rate (HR [1/min]).

The values of these parameters will be recorded beat-to-beat. Specific calculations conducted, as part of further analysis, will be based on the values obtained in the supine position.

The ICG examinations will be done before AFR and during each follow up visit.

Invasive right heart catheterization. All eligible patients before enrolling to the study will undergo a diagnostic right heart catheterization using a Swan-Ganz catheter (SGC). After ultrasound-guided puncture of internal jugular or subclavian vein, a SGC will be introduced via the right ventricle to a pulmonary artery. The following pressure curves will be obtained in patients at rest:

- Right atrium/upper vena cava pressure;

- Right ventricle pressure;

- Mean pulmonary artery pressure;

- Pulmonary artery wedge pressure (PAWP).

Furthermore, a cardiac output (CO) will be measured using the thermodilution method.

The following hemodynamic parameters will be calculated:

- Cardiac index;

- Systemic vascular resistance;

- Pulmonary vascular resistance.

In the next step, subjects will be asked to perform a handgrip test for a maximal tolerated period of time until fatigued. The measurement of PAWP and $\mathrm{CO}$ will then be obtained.

If a resting PAWP will be above $15 \mathrm{mmHg}$ or exercise PAWP above $25 \mathrm{mmHg}$, patients will be qualified for AFR implantation. A right atrial pressure above $20 \mathrm{mmHg}$ or exceeding PAWP will be the contraindication for the atrial shunting procedure.

Invasive catheterization will be performed before AFR and during the first follow up visit.

The AFR procedure. The procedure will be performed under general anesthesia. A threedimensional TEE guided trans-septal puncture will be performed followed by insertion of an Amplatzer Stiff wire in a left upper pulmonary vein. Next, a 10-14 mm diameter balloon septostomy to facilitate AFR implantation through a $12-14 \mathrm{~F}$ dedicated delivery system. Before releasing the device, an invasive trans atrial gradient will be measured through an 8-10 $\mathrm{mm}$ fenestration. The left to right shunt and mean gradient will be confirmed in TEE. After releasing an AFR device, a delivery system will be removed and a puncture site will be sealed with hemostatic suture. 
The patients will be discharged during the following couple of days. Subjects with history of atrial fibrillation will continue anticoagulation therapy and those without atrial fibrillation will be treated with double antiplatelet drugs for 3 months.

\section{Outcomes}

Primary outcome measures. The primary outcome is clinical improvement within 12 months after AFR implantation defined as at least $10 \%$ increase in six-minute walk test (6MWT) compared to baseline.

Secondary outcomes measures. (1) Clinical improvement expressed in reduction of New York Heart Association (NYHA) class; (2) Device related adverse event: device migration, embolization, device related thrombus, shunt occlusion, or need for device removal.

Other outcome measures. (1) Reduction of PAWP at rest 30 days after AFR implantation.

(2) Reduction of PAWP during a handgrip test 30 days after AFR implantation. (3) Kansas City Cardiomyopathy Questionnaire (KCCQ-12) within 12 months after AFR implantation. (4) Clinical adverse events within 12 months after AFR implantation: cardiac mortality or rehospitalization for HF decompensation.

Correlation of echocardiographic, invasive and impedance parameters with clinical outcome. All hemodynamic data will be analyzed post-hoc to test any correlation with clinical outcome after AFR procedure.

\section{Data collection and management}

All clinical data including demographic information, results of blood laboratory tests performed locally will be stored for further analysis. Digital files containing raw data obtained from echocardiography and fluoroscopy will also be collected.

\section{Statistical methods}

Continuous data will be summarized by means of mean value, median, minimum, maximum, standard deviation and number of observations. Categorical data will be expressed as means of absolute and relative frequencies. A series of statistical tests including: the Shapiro-Wilk test, ANOVA, unpaired t-test, the Mann-Whitney $\mathrm{U}$ or $\chi^{2}$ test will be done. Clinical adverse events will be tabulated.

\section{Ethical considerations}

A local ethics committee has approved the PROLONGER study protocol.

\section{Discussion}

The concept of creating an interatrial shunt leading to decompression of left atrium is an attractive therapy for patients with HF.

Presented herein, is the PROLONGER study protocol. This is a single center, open label clinical study aimed at defining invasive and non-invasive clinical parameters that could predict a positive response for implantation of AFR.

Three available shunt devices have been tested in a series of small studies to date. The initial results are promising; however, the question of which population would benefit most from interatrial shunting has been raised.

In the AFR PRELIEVE study some, but not all patients showed symptoms improvement. The 6MWT distance significantly increased only among HFrEF patients. The interpretation of single statistically significant results such as change in NYHA class or increase in 6MWT distance should be cautious, because the patient numbers were small. Both HFpEF and HFrEF patients presented reduced resting PAWP compared to baseline, however only in HFpEF subjects, the difference reached statistical significance [8].

In the present study we are going to focus on clinical parameters as: 6MWT test and NYHA functional class.

Although the study is not designed to show device related adverse events, these will also be reported.

A hemodynamic profile will be assessed by an invasive right heart catheterization as well as using non-invasive impedance cardiography.

The ICG is a non-invasive diagnostic tool enabling reliable assessment of patients' hemodynamic profile [9-13].

There are data supporting a good correlation between impedance diagnostics and invasive assessment of cardiac output and systemic vascular resistance [14]. Our previous experience with this procedure in patients with acute myocardial infarction was recently published [15].

Unlike previously published studies, the focus will be to perform exercise invasive catheterization using a simple handgrip test instead of standard cyclo-ergometer. Although a handgrip test in not well validated, it allows a patient to perform significant exertion.

The hemodynamic data obtained from SGC and ICG will be analyzed post-hoc in order to seek any correlation of cardiac filling pressures and cardiac performance with clinical improvement after the AFR procedure. 
The first patient enrolled to PROLONGER study presented substantial clinical improvement in the 6MWT distance, which almost doubled and a reduction of NYHA class. Diagnostic right heart catheterization revealed a significant reduction in PAWP, mean pulmonary artery and right atrial pressures [16].

A 12 month follow-up is planned with four control visits, which should allow assessment of clinical and hemodynamic responses for AFR.

\section{Conflict of interest: None declared}

\section{References}

1. Mosterd A, Hoes AW. Clinical epidemiology of heart failure. Heart. 2007; 93(9): 1137-1146, doi: 10.1136/hrt.2003.025270.

2. Redfield MM, Jacobsen SJ, Burnett JC, et al. Burden of systolic and diastolic ventricular dysfunction in the community: appreciating the scope of the heart failure epidemic. JAMA. 2003; 289(2): 194-202, doi: 10.1001/jama.289.2.194, indexed in Pubmed: 12517230.

3. Ponikowski P, Voors A, Anker S, et al. 2016 ESC Guidelines for the diagnosis and treatment of acute and chronic heart failure. Eur Heart J. 2016; 37(27): 2129-2200, doi: 10.1093/eurheartj/ehw128.

4. Lam CSP, Voors AA, de Boer RA, et al. Heart failure with preserved ejection fraction: from mechanisms to therapies. Eur Heart J. 2018; 39(30): 2780-2792, doi: 10.1093/eurheartj/ehy301, indexed in Pubmed: 29905796.

5. Borlaug BA, Paulus WJ. Heart failure with preserved ejection fraction: pathophysiology, diagnosis, and treatment. Eur Heart J. 2011; 32(6): 670-679, doi: 10.1093/eurhearti/ehq426, indexed in Pubmed: 21138935.

6. Shah SJ, Feldman T, Ricciardi MJ, et al. One-Year safety and clinical outcomes of a transcatheter interatrial shunt device for the treatment of heart failure with preserved ejection fraction in the reduce elevated left atrial pressure in patients with heart failure (REDUCE LAP-HF I) trial: a randomized clinical trial. JAMA Cardiol. 2018; 3(10): 968-977, doi: 10.1001/jamacardio.2018.2936, indexed in Pubmed: 30167646.

7. Guimarães L, Bergeron S, Bernier M, et al. Interatrial shunt with the second-generation V-Wave system for patients with advanced chronic heart failure. EuroIntervention. 2020; 15(16):
1426-1428, doi: 10.4244/EIJ-D-19-00291, indexed in Pubmed: 31422927.

8. Paitazoglou C, Özdemir R, Pfister R, et al. The AFR-PRELIEVE trial: a prospective, non-randomised, pilot study to assess the Atrial Flow Regulator (AFR) in heart failure patients with either preserved or reduced ejection fraction. EuroIntervention. 2019; 15(5): 403-410, doi: 10.4244/EIJ-D-19-00342, indexed in Pubmed: 31130524.

9. Niu X, Zhang Q, Xiao D, et al. A retrospective study of hemodynamic changes in patients after off-pump coronary artery bypass graft surgery using impedance cardiography. Med Sci Monit. 2019; 25: 3454-3462, doi: 10.12659/MSM.913289, indexed in Pubmed: 31073116.

10. Louvaris Z, Spetsioti S, Andrianopoulos V, et al. Cardiac output measurement during exercise in COPD: A comparison of dye dilution and impedance cardiography. Clin Respir J. 2019; 13(4): 222-231, doi: 10.1111/crj.13002, indexed in Pubmed: 30724023.

11. Małek ŁA, Mróz A, Czajkowska A, et al. Accuracy of impedance cardiography for hemodynamic assessment during rest and exercise in wheelchair rugby players. Res Q Exerc Sport. 2019; 90(3): 336-343, doi: 10.1080/02701367.2019.1600651, indexed in Pubmed: 31082312.

12. Kurpaska M, Krzesiński P, Gielerak G, et al. Exercise impedance cardiography reveals impaired hemodynamic responses to exercise in hypertensives with dyspnea. Hypertens Res. 2019; 42(2): 211-222, doi: 10.1038/s41440-018-0145-y, indexed in Pubmed: 30504821.

13. Woltjer HH, Bogaard HJ, de Vries P. The technique of impedance cardiography. Eur Heart J. 1997; 18(9): 1396-1403, doi: 10.1093/ oxfordjournals.eurheartj.a015464.

14. Silver MA, Cianci P, Brennan S, et al. Evaluation of impedance cardiography as an alternative to pulmonary artery catheterization in critically ill patients. Congest Heart Fail. 2004; $10(2$ Suppl 2): 17-21, doi: 10.1111/j.1527-5299.2004.03410.x, indexed in Pubmed: 15073481.

15. Lewicki L, Fijalkowska M, Karwowski M, et al. The non-invasive evaluation of heart function in patients with an acute myocardial infarction: The role of impedance cardiography. Cardiol J. 2019 [Epub ahead of print], doi: 10.5603/CJ.a2019.0098, indexed in Pubmed: 31642052.

16. Lewicki $€$, Sabiniewicz R, Siebert J, et al. Atrial flow regulator as a novel therapy for patients with chronic heart failure. Cardiol J. 2020; 27(3): 309-311, doi: 10.5603/CJ.a2020.0077, indexed in Pubmed: 32436584. 